\title{
Photophysical Studies on the Mono- and Dichromophoric Hemicyanine Dyes I. Photoelectric Conversion from the Dye Modified ITO Electrodes
}

\author{
Yanyi Huang, Tianrong Cheng, Fuyou Li, and Chun-Hui Huang* \\ State Key Laboratory of Rare Earth Materials Chemistry and Applications, and PKU-UHK Joint Lab on Rare \\ Earth Materials and Bioinorganic Chemistry, College of Chemistry, Peking University, Beijing 100871, China
}

\author{
Tingjun Hou, Anchi Yu, Xinsheng Zhao, and Xiaojie Xu \\ Department of Chemistry, College of Chemistry, Peking University, Beijing 100871, China
}

Received: April 3, 2002

\begin{abstract}
Stilbazolium dimers were designed and synthesized in which methylene groups of different lengths link the two chromophores. The second-order nonlinear optical property has been detected from their LangmuirBlodgett monolayers. Photocurrent generation as well as other photophysical properties such as absorption and fluorescence were investigated. Comparing the dimers with the stilbazolium monomer, we found strong second harmonic generation (SHG) signals and remarkable enhanced photoelectric conversion (PEC) quantum yields from 1,3-Bis [(E)- 4-(2-(4-( $N$-methyl, $N$-octadecylamino) phenyl) ethenyl) pyridinyl]-alkane dibromide (B3) and 1,5-Bis [(E)- 4-(2-(4-( $N$-methyl, $N$-octadecylamino) phenyl) ethenyl) pyridinyl]-alkane dibromide (B5). The structures of the Langmuir-Blodgett films of these dyes are assumed. Although these dimers are center-symmetric (or quasi-center-symmetric) molecules, they can form the ordered non-center-symmetric (in normal direction) monolayer by the compress force and the molecular interaction between the amphiphilic dye molecules and the water molecules at the air/water interface. The influences of bias, donor/acceptor, soluble oxygen, and light intensity to photocurrent generation were studied; our results provide a new perspective to improve the performance of functional molecular material without any change of the chemical structure of the chromophore itself.
\end{abstract}

\section{Introduction}

Organic molecules, which have promising functions for optics and electronics, are important in various imaginable applications. Stilbazolium (hemicynane) and its derivatives have been studied for several decades because of their potential applications to molecular-based functional materials in opto-electric fields. Their photophysical properties, that is, absorption, fluorescence, nonlinear optical phenomena (second harmonic generation (SHG), two-photon absorption, laser application), and photoelectric conversion (PEC) have been widely reported. ${ }^{1-14}$ As one of the most efficient SHG chromophores, it has many derivatives that have been designed and synthesized to improve its SHG property. ${ }^{15,16}$ Stilbazolium is also a useful chromophore as a voltage-sensitive membrane probe, especially used in cell biology. ${ }^{17}$ The photophysical properties of stilbazolium have been investigated for years both in theoretical and experimental regions, and there are still numerous reports on the details of the picture of photophysics. The most important feature of stilbazolum is that photoexcitation induces an intramolecular charge-transfer excited state. This still guides our research on stilbazolium and its derivatives.

In recent years, molecular aggregates and many molecular dimers have been synthesized to study the differences between their novel photophysical properties and those of individual monomers. ${ }^{18-23}$ Results show that the interaction between the monomers (chromophores) in those dimers and aggregates can change their photophysical behaviors. But rarely are there reports

* To whom the correspondence should be addressed. on the potential value of these phenomena. For years, we have devoted ourselves to find effective chromophores for nonlinear optical and/or photoelectric conversion applications. In the past several years, we have reported several papers on the photoelectric conversion of stilbazolium and its derivatives. ${ }^{24-31} \mathrm{We}$ have found that the chemical modification (even a small change of the group's position) of the chromophore can tremendously alter its photophysical properties and functions, especially for those properties related to charge distribution (or charge transfer). A special modification of functional material is molecular dimerization, through which, as we have reported, some photophysical functions will be improved remarkably. ${ }^{24,25}$ We find that a simple dimerization, in which the functional chromophore is not changed at all and the charge distribution is almost the same as in monomer, can make a huge difference. In this article, we attempt to show the relationship between the structures and properties of the molecules through an example of a series of stilbazolium dimers.

Here, we synthesize a series of stilbazolium dimers: B3, B5, and B12 (Figure 1) and report their photophysical properties, especially the SHG from LB monolayer and their PEC abilities from LB films modified ITO electrodes. Both of these phenomena are related to charge redistribution upon the light excitation. The strong SHG signal indicates that the molecular orientation in LB film should not be the center-symmetric. We assumed that the actual conformation of dimers will be a "folded shape". Another important improvement of stilbazolium's photoactive properties is that the photoelectric conversion quantum yields of some dimers are greatly enhanced by 


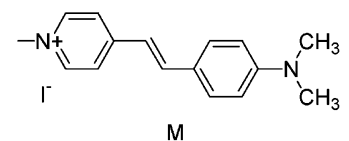

M
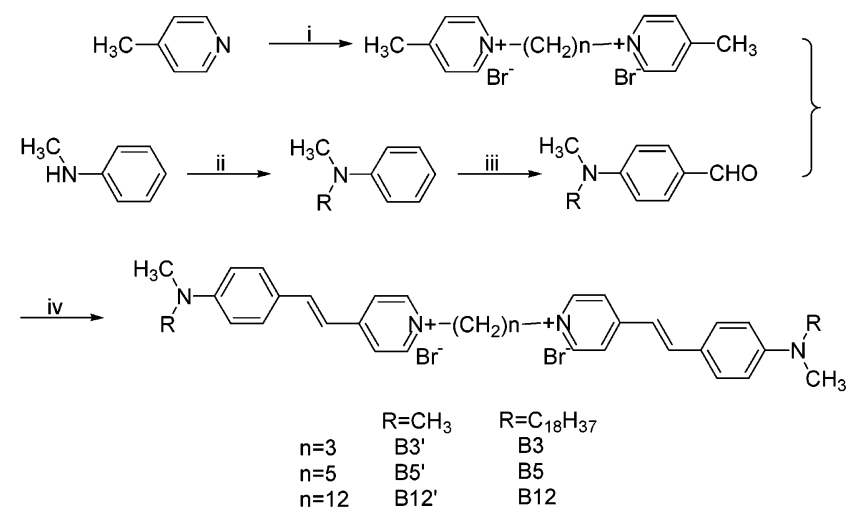

Figure 1. Molecular structures and schematic synthesis processes of dyes. (i) 1,n-dibromoalkane, anhydrous ethanol, reflux; (ii) alkyl iodide, toluene, $\mathrm{NaOH}$, cat.; (iii) $\mathrm{POCl}_{3}$, DMF, reflux; (iv) anhydrous ethanol, reflux, cat.

dimerization with suitable alkyl chains. Most reports on molecular dimers ${ }^{21-23}$ are related on the dramatically steady-state spectroscopic difference of the chromophore after dimerization. However, in our system, the steady-state absorption spectra between dimers and monomers are not very different from each other, neither are their emission spectra. This has not happened to many dimers reported before. ${ }^{21-23}$ Having observed the interesting relationships between their structures and the photophysical properties, we propose that many property changes after dimerization cannot be simply explained by the effect on the intramolecular aggregation. The origin of these property changes caused by dimerization may be the effect of dynamic photophysical properties and should be pursued.

\section{Results and Discussions}

1. Molecular Design and Synthetic Procedures. The structures of molecules of monomers and dimers, as well as their schematic synthetic procedures are listed in Figure 1. Details of the synthetic procedure and structure identification can be found in the Experimental Section. $M$ is a common stilbazolium molecule (monomer), which has been investigated thoroughly in nonlinear optical and molecular electrical fields. In this article, $\mathrm{M}$ is used as a reference material to evaluate the properties of other new materials. $\mathrm{B} n(n=3,5$, and 12) represents those designed bichromophore stilbazolium molecules (dimers), and $n$ is the number of methylene groups by which two chromophores are linked together. The attempts to synthesize these dimers are (1) to investigate the SHG from a centersymmetric (or quasi-center-symmetric) molecule, which can be changed to a noncenter-symmetric conformation by external force field; (2) to explore the relationship between the photoelectric conversion properties and the molecular structure; and (3) to improve the film stability with dimerization. Also, the different lengths of linkage are designed to control the distance between two chromophores in dimer molecules. The 3-methylene-group is a commonly used linkage for most dichromophoric molecules to induce two chromophores to align in a parallel fashion and form an excimer; ${ }^{29}$ whereas the $12-$ methylene-group is long enough to make two chromphores separated to individual under relative free condition such as in solution phase.

The major synthetic steps are marked in Figure 1. They are modified from refs 32,33 .

Two series of similar bichromophoric dyes B3, B5, B12, and B3', B5', B12' have been synthesized (see the Experimental Section for details). The only difference in the alkyl-amino groups is that the former series involves long-tail (octadecayl-) molecules, whereas the latter involves short-tail (methyl-) ones. The two series of molecules are designed for different usages, the long-tail amphiphilic ones being employed for monolayer formation, whereas the short-tail ones being easily dissolved in most polar solvents for solution investigations.

Figure 2 shows the ${ }^{1} \mathrm{H}$ NMR spectra of $\mathrm{M}^{\prime}, \mathrm{B} 3^{\prime}, \mathrm{B}^{\prime}$, and $\mathrm{B} 12^{\prime}$ in the region $\delta 6.5-9 \mathrm{ppm}$. This region contains those chemical shifts of hydrogen atoms in benzene ring, pyridinyl ring, and the central double bond. All of the peaks are doublets, and each peak is assigned to the corresponding hydrogen atoms in molecule, as shown in Table 1. More details of all of the Dyes' chemical shifts and the coupling constants are also listed in Table 1. The most important common feature of the NMR data is that the coupling constants of $c$ and $d$ are all around 16 Hz. This value indicates that all dyes are trans-isomers. Moreover, the spectra of B3, B5, and B12 (or B3', B5', and $\left.\mathrm{B} 12^{\prime}\right)$ are almost the same in the region $\delta 6.5-9 \mathrm{ppm}$. The spectra of dimers is quite similar to the spectrum of monomers, with the exception that the chemical shift of $a$ in a monomer is obviously less than that found in dimers. This difference is surely caused by those different substituent linked to $\mathrm{N}$ atom of pyridinyl ring because the methyl group in $\mathrm{M}$ has a weaker electron-pushing induction effect than that of the longer alkyl chains in dimers. Furthermore, the induction effect is not valid
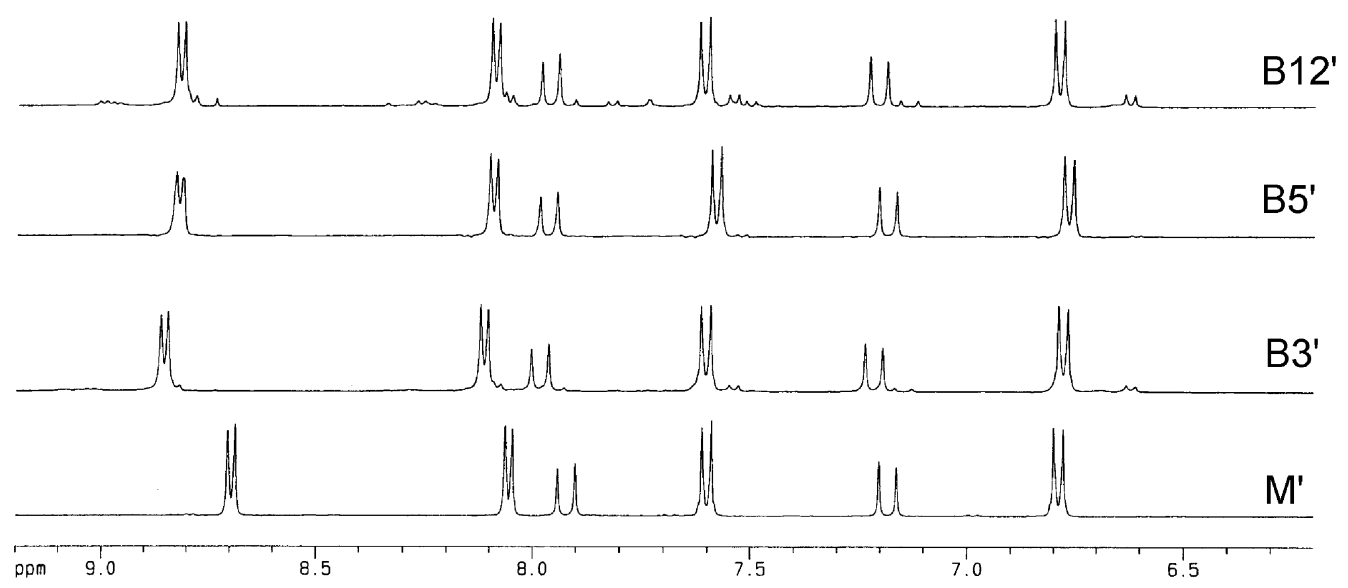

Figure 2. ${ }^{1} \mathrm{H}$ NMR spectra of $\mathrm{M}^{\prime}, \mathrm{B} 3{ }^{\prime} \mathrm{B} 5^{\prime}$ and $\mathrm{B} 12^{\prime}$ in DMSO. 


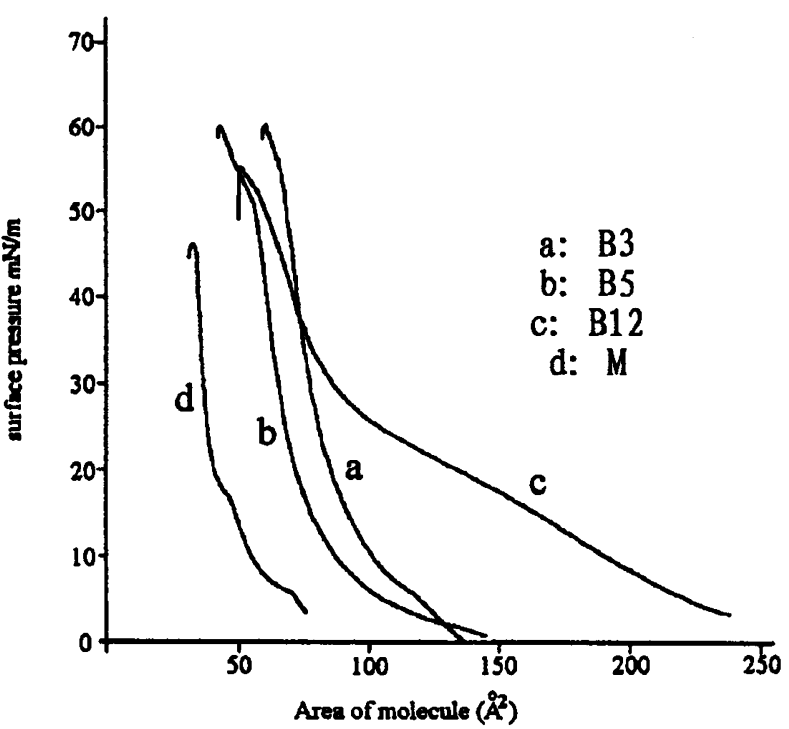

Figure 3. Surface pressure $(\pi)$-area per molecule (A) isotherms of dyes. (a) B3, (b) B5, (c) B12, and (d) M.

TABLE 1: NMR Chemical Shifts and Coupling Constants of Dyes

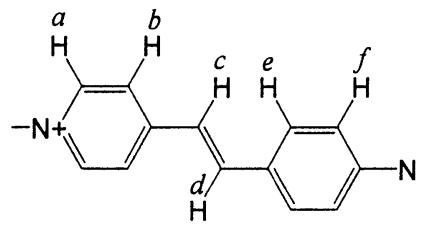

\begin{tabular}{|c|c|c|c|c|c|c|c|c|}
\hline \multirow[b]{2}{*}{ DMSO } & \multicolumn{8}{|c|}{ chemical shift $(\delta, \mathrm{ppm})$ [coupling constant $(\mathrm{J}, \mathrm{Hz})]$} \\
\hline & \multicolumn{2}{|r|}{$\mathrm{M}^{\prime}$} & \multicolumn{2}{|c|}{ B3' $^{\prime}$} & \multicolumn{2}{|r|}{$\mathrm{B}^{\prime}$} & \multicolumn{2}{|c|}{ B12' } \\
\hline$a$ & 8.70 & [6.84] & 8.85 & [6.82] & 8.82 & [6.80] & 8.82 & {$[6.9$} \\
\hline$b$ & 8.06 & [6.88] & 8.12 & [6.92] & 8.09 & [6.69] & 8.09 & 6.9 \\
\hline$c$ & 7.92 & [16.12] & 7.98 & [16.02] & 7.97 & [16.06] & 7.96 & {$[16.0$} \\
\hline$d$ & 7.18 & [16.12] & 7.22 & [16.04] & 7.19 & [16. & 7.21 & {$[16.0$} \\
\hline$e$ & 7.60 & [8.88] & 7.60 & [8.92] & 7.58 & {$[8$.} & 7.61 & {$[8.9$} \\
\hline$f$ & 9 & {$[8$.} & 6.78 & [8.97] & 6.77 & [8.90] & 6.79 & {$[9.0$} \\
\hline $\mathrm{CDCl}_{3}$ & \multicolumn{2}{|c|}{ M } & \multicolumn{2}{|c|}{ B3 } & \multicolumn{2}{|c|}{ B5 } & \multicolumn{2}{|c|}{ B12 } \\
\hline$a$ & 74 & {$[6$} & 9.34 & 6. & 9. & & & \\
\hline$b$ & 7 & & 7.69 & [6 & 7.8 & & 7.8 & \\
\hline$c$ & 7.60 & [15.92] & 7.54 & {$[15.8$} & 7.65 & {$[15.86]$} & 7.64 & {$[15$.} \\
\hline$d$ & 6.85 & {$[15.8$} & 6.80 & {$[15.8$} & 6.91 & {$[15$.} & 6.87 & {$[15$.} \\
\hline$e$ & 7.53 & [8.96] & 7.49 & [8.95] & 7.58 & [8.89] & 7.52 & \\
\hline$f$ & 6.66 & $0.90]$ & 6.67 & 0] & 6.75 & 8.59] & 6.66 & \\
\hline
\end{tabular}

beyond 4 chemical bonds, so that there is almost no difference between the electron-pushing abilities of those alkyl linkages in dimers. For this reason, the NMR spectra of dimers are extreme similar. This similarity indicates that the dimerization has not influence the structure of stilbazolium chromophore.

2. Structures of Langmuir-Blodgett Films and Their Second Harmonic Generation. The Langmuir-Blodgett film formation and its structure are of considerable importance for the final functional performance because our SHG signal will closely relate to the film structure, and our evaluating device is based on an LB monolayer modified ITO electrode.

The surface pressure $(\pi)$ - molecular area (A) isotherms of the long-tail dyes are shown as Figure 3. It is quite clear that all of these amphiphilic molecules are capable of forming stable and rigid Langmuir films at air/water interface. Also, the collapse pressures of these four molecules (B3, B5, B12, and $\mathrm{M})$ indicate that the dimerization can improve the stability of the Langmuir monolayer. There is no doubt that the orientation of the molecules would be like this: the long alkyl tails
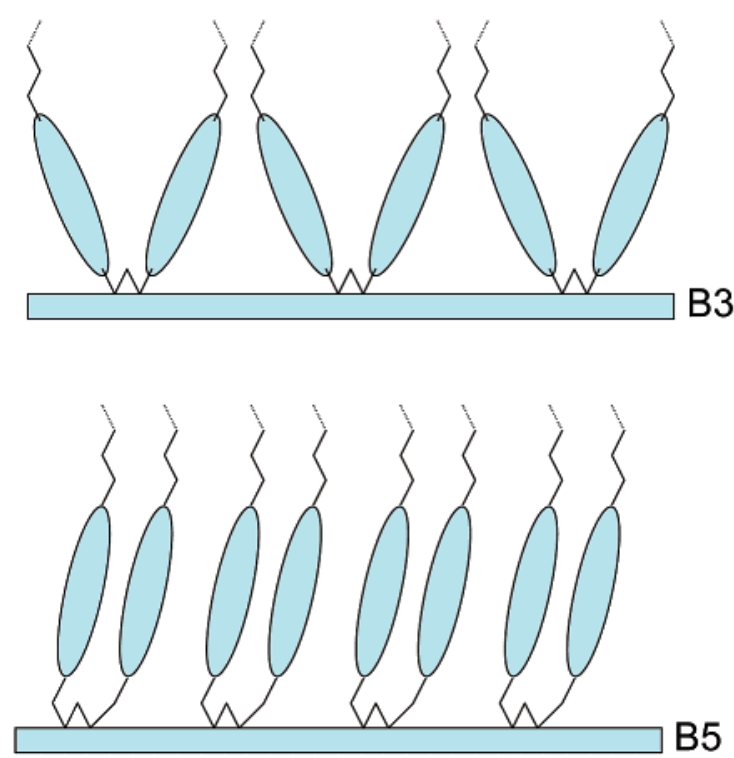

Figure 4. Possible conformation of B3 (a) and B5 (b) molecules in LB films.

TABLE 2: Molecular Limiting Areas and Collapse Pressures of Stilbazolium Dyes

\begin{tabular}{lcccc}
\hline & M & B3 & B5 & B12 \\
\hline $\begin{array}{l}\text { molecular limiting } \\
\begin{array}{l}\text { area }\left(\mathrm{nm}^{2}\right) \\
\text { collapse pressure } \\
(\mathrm{mN} / \mathrm{m})\end{array}\end{array}$ & 0.40 & 0.90 & 0.80 & 1.20 \\
& 46 & 60 & 60 & 55
\end{tabular}

(hydrophobic parts) point into the air, whereas the most hydrophilic sites are partly dip into the water. The substrates for LB film deposition are freshly pretreated by a Piranha solution (for quartz) or sodium methate (for ITO), which produces very good hydrophilic surfaces. Thus, the LB films should have orientations similar to the floating Langmuir films. Table 2 provides the values of the molecular limiting area of these four molecules. It is worth noting that the molecular limiting area of B3 is even larger than that of B5. We assume that this occurs because the B5 molecule can form a folded conformation whose two chromophores in one molecule are nearly parallel, whereas B3 cannot. Under the balance of the different forces (compression force, the hydrophobic-hydrophobic interaction between the long alkyl chains in LB films and the static electric repulsion between the pyridium moieties), the B5 is tightly stacked, and the area it occupies is exactly twice that of $\mathrm{M}$, indicating the package structure of stilbazolium chromophores in the LB film of B5 is quite similar to that in the LB film of M. Although a three-methylene-chain is usually suitable for linking two parallel chromophores, ${ }^{29}$ B3 is an exception. The static electric repulsion between the partially localized positive charges on pyridium moieties of the two chromophores in B3 separates them, whereas the three-methylene chain is not long enough for these two charged moieties to be parallel. Hence, Figure 4 can be used to illustrate our hypothesis. In the LB film, the B3 molecule has a "V-shape" conformation and this causes a larger occupation area compared with B5 which is a "U-shape" folded molecule. Because the linkage between the two chromophores in B12 is too long (12 methylene groups) and its conformation can hardly be demonstrated, its image is not shown here. But it is obvious that B12 occupies more space than others because the twelve-methylenelinkage is closed to hydrophilic sites and occupies some space when the film is deposited onto a hydrophilic surface. 
TABLE 3: Results of SHG of Dyes' LB Films

\begin{tabular}{lrrrr}
\hline & M & B3 & B5 & B12 \\
\hline$\chi_{\text {zzz }}\left(\mathrm{pm} \mathrm{V}^{-1}\right)$ & 216 & 148 & 207 & 173 \\
$\chi_{\text {zxx }}\left(\mathrm{pm} \mathrm{V}^{-1}\right)$ & 33 & 60 & 40 & 19 \\
$\phi(\mathrm{deg})$ & 29 & 42 & 32 & 25
\end{tabular}

The LB monolayers' SHG signals support our hypothesis very well. The measurement is taken by means of a standard LB film method, ${ }^{30}$ and the results are shown in Table 3 . The value of can be calculated by effective nonlinear coefficient, and the latter is measured by comparing the SHG signal of the sample with the Y-cut quartz. It is found that the SHG signal of B5 is as strong as that of monomer, and the fact that $\chi_{\text {zzz }}$ values from B3, B12 LB films are also comparable with $\mathrm{M}$ indicates that the molecular orientation in the LB films of dimers should be a folded shape and noncenter-symmetric. Ashwell et al. ${ }^{31}$ has reported an example of the SHG from the LB films of centersymmetric squaraine compounds to form non symmetric structure in LB films through the interaction between molecules. Here, our results provide another example that these dimer molecules, which can form nonsymmetric conformations in LB films through intramolecular folding, to give strong SHG signals. Furthermore, these dimers should provide higher monolayer stability than monomer.

The average tilt angle $\phi$ is the angle between the surface normal and the dipole of the chromophore; it can be calculated from eq $1^{34-36}$

$$
\tan \phi=\left[\left(\frac{I_{2 \omega}^{p-p}}{I_{2 \omega}^{s-p}}\right)^{1 / 2}-\frac{3}{2}\right]^{-1 / 2}
$$

where $I$ stands for the intensity of output light beam, subscript $2 \omega$ represents the second harmonic generation output, and superscript $p-p$, and $s-p$ represent the relationship between the polarities of input and output light beams. Here, we find that the average tilt angles of the dipole of $\mathrm{M}, \mathrm{B} 5$, and $\mathrm{B} 12$ are all about $30^{\circ}$, whereas the tilt angle of the dipole of $\mathrm{B} 3$ is about $40^{\circ}$, indicating that compared with the LB films of other dyes the chromophores in the B3 LB film are less perpendicular to the substrate surface. It is evident that if all of the dipoles in one LB film are nearly parallel (tilted to the same direction), the LB film will show strong optical nonlinear behavior; whereas if the directions of the dipoles are not parallel, the chromophore's optical nonlinear ability cannot be summed up together effectively. The $\chi_{\text {zzz }}$ values in Table 3 give us the clear idea that in B3 LB film the parallelity of chromophores is not as strong as that in $\mathrm{M}$ or B5 LB films, because the $\chi_{\mathrm{zzz}}$ value of B3 LB film is much smaller than that of M or B5 LB films. As Figure 4 shows, the results of tilt angle and of the $\chi_{\text {zzz }}$ value both strongly support our interpretation of the structure of LB films. As for the B12 LB film, that the optical nonlinear signal is smaller than M or B5 is readily explained by the less-ordered film structure, which is caused by the long linkage moiety.

One may ask whether the five-methylene-linkage in B5 molecule is long enough for the two positive charged chromophores to be parallel. The single-crystal structure of B5' $^{\prime}$ supports our point of view that B5 can form a "U-shape" conformation to make the two chromophores nearly parallel, even though the attempts to get single crystals of B3, B5, B12, and B3', B12' have been finally failed. The only difference between $\mathrm{B} 5^{\prime}$ and $\mathrm{B} 5$ is that the long alkyl tail in B5 molecule is substituted by methyl. The good single crystals of B5' with dimensions ca. $0.6 \times 0.4 \times 0.3 \mathrm{~mm}^{3}$ was obtained from the mixture solvent of water and ethanol. However, because most

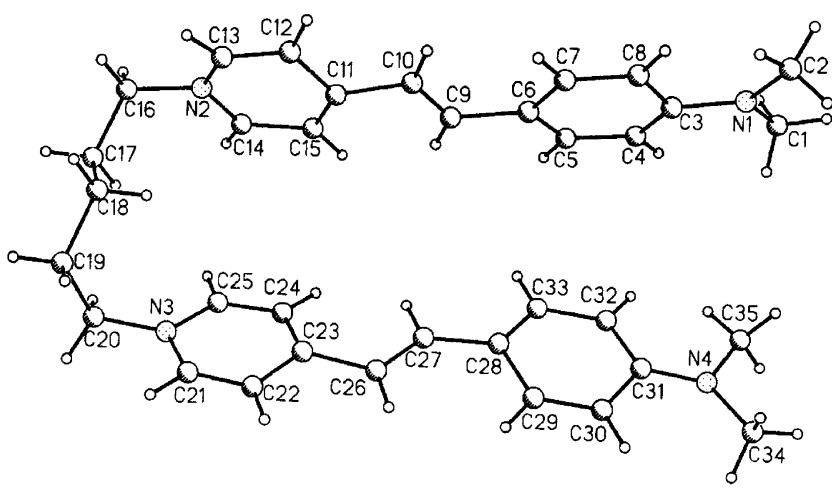

Figure 5. Crystal structure of the cationic fragment of $\mathrm{B}^{\prime}$.

of the samples are too ephemeral under the exposure of X-rays, the structure determination by X-ray diffraction becomes very difficult. Finally, there is enough data collected from one crystal sample. Although the data set is not extensive enough for a detailed evaluation of those anisotropic displacement coefficients for all atoms, the framework of the molecule is clear, as shown in Figure 5: even when there is no compressive force, $\mathrm{B}^{\prime}$ tends to form a folded-shape conformation and the two chromophores are nearly parallel.

The results of molecular mechanical calculations also support our assumption of molecular structures in LB films. All of the structures used in this computational section are short-tail derivatives, to reduce the difficulty in computation. Conformation searching (cvff95 field force) reveals that the most favorable conformation of B3 is an "extended" one (Figure 6(a)); whereas B5, a "folded" conformation (Figure 6(b)), which is very similar to the result of the crystal structure. This difference is consistent with the discussion on the limiting area of occupancy of these molecules and tilt angles get from SHG results. Obviously, even though the $\pi-\pi$ stacking might exist in the dimers between the two $\pi$-conjugated chromophores, the static electric repulsion between the two positive charged chromophores of B3 molecule induces their nonparallelity.

There is another possible reason for the difference between the dimers and the monomer: the intrinsic values of dimers' chromophores might be changed by intramolecular coupling due to dimerization. According to the successful model drawn by Oudar et al. ${ }^{37,38}$ that describes the organic chromophores's NLO activity

$$
\beta=\frac{3 e^{2}}{2 \hbar^{2}} \frac{\omega_{\mathrm{ge}} f_{\mathrm{ge}} \Delta \mu_{\mathrm{ge}}}{\left(\omega_{\mathrm{ge}}^{2}-\omega^{2}\right)\left(\omega_{\mathrm{ge}}^{2}-4 \omega^{2}\right)}
$$

where $\omega$ is the irradiation frequency, $\omega_{\mathrm{ge}}$ is the frequency between the ground (g) and excited (e) states; $f$ is the oscillation strength; and $\Delta \mu_{\mathrm{ge}}$ is the change of the dipole moment before and after the light irradiation. The same chromophores should have the same changes in dipole moments $(\Delta \mu)$ under light irradiation if the electronic states have not been modified by outer fields. In our system, if the intramolecular coupling is existed in the dimer molecules, it will surely affect the electronic structure of the energy states of chromophores and then influence the NLO abilities of molecules and also the other photoactive properties, such as absorption and emission. However, as we will point out later, the absorption spectra indicate a very light intramolecular coupling of dimers; therefore, we take structureeffect as the major factor to influence the molecular/chromophore's hyperpolarizability.

In summary, the dimers hold the folded conformations in LB films and the chromophore orientations of dimers are similar 

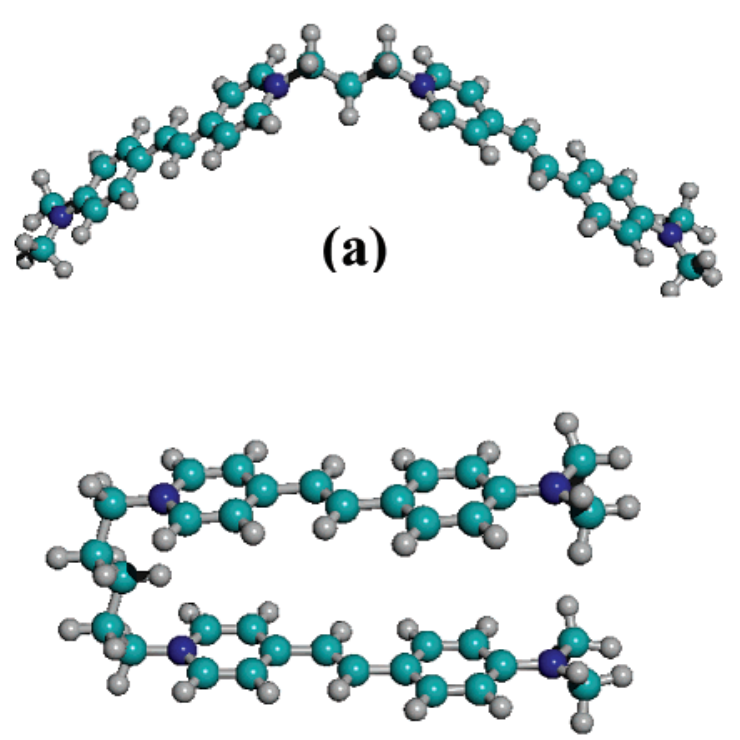

(b)

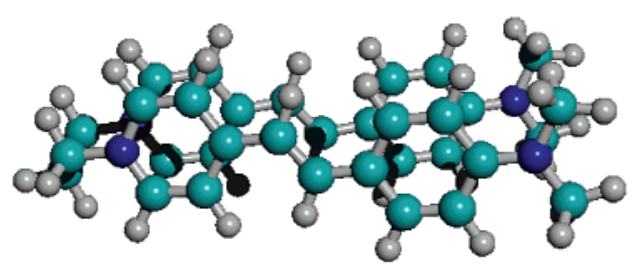

(c)

Figure 6. Most stable conformation of B3 (a) and B5 (b), and the model conformation of a folded B3, named "B3f" (c).

to that of M. But evidently, the structures of the dyes' LB films are different from each other, and this difference might bring changes to the photophysical properties of molecules.

3. Steady-State Spectroscopic Properties and Photocurrent Generation. Absorption spectra always reflect molecular interactions, especially those between two or more chromophores. If there are molecular aggregates in the system, then the absorption spectroscopic method is almost the most powerful technique for identifying them. The absorption spectra of dyes are shown in Figure 7: (a) is for the spectra of chloroform solutions while (b) for 1-layer LB films. Evidently, there is no dramatic difference between the solution spectra of the dyes. The shift between the dimers and the monomer is very slight in major peaks around $480 \mathrm{~nm}$. We cannot observe the absorption shift when the concentration of the solution changes. That is to say, in solution, the aggregation can be ignored. Our result of monomer in LB film shows that the aggregation can also be ignored under our experimental conditions. Song et al. ${ }^{6}$ have investigated the aggregation of hemicyanine molecule, and their results show that for some derivatives the formation of aggregates is very slow at the air/water interface. As we know, the aggregation process of hemicyanine derivatives can be dramatically influenced by chemical modification. If the langmuir films are deposited onto the substrates once the surface pressures reach our pre-set values, the LB films will be nonaggregate dominant. For the monomer molecule in our work, prolonging the compressing procedure will increase the proportion of aggregate in LB films; meanwhile, the dimer molecules show the same behavior. These phenomena are similar to the
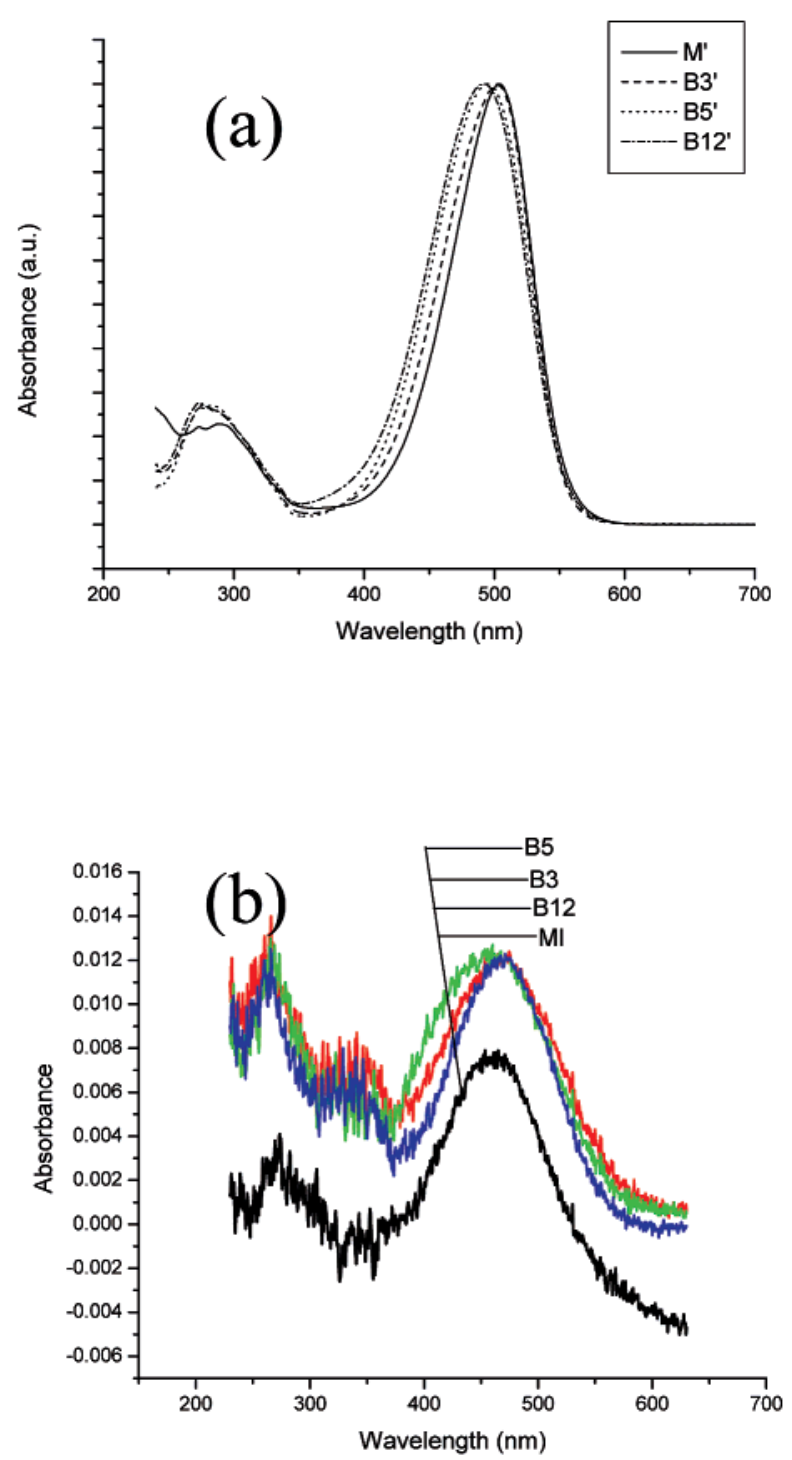

Figure 7. Absorption spectra of dyes. (a) chloroform solutions; (b) one-layer LB films on quartz substrates.

reports of Bohn et al. ${ }^{9,39}$ Because it is difficult to precisely control the formation of a certain amount of aggregates of the LB films, we have not investigated the details of those dimers' photocurrent generation and related photochemistry. Instead, what we are interested is the monomer-like absorption band in the visible-light region, not the aggregates' absorption band in the UV region. Under the same experimental conditions in our work, the films of different molecules are all controlled to be nonaggregate dominant, and the photofunctions are all from the monomers/isolated molecules. Another supportive evidence is that the SHG signal intensities of the dimer molecules are comparable with that of the monomer and all signals are from the nonaggregates because the aggregate will strongly reduce the SHG signal intensity. ${ }^{40-42}$

From Figure 7(b), we see only a minor difference between the dimers and monomer: all dimers have a weak absorption peak around $330 \mathrm{~nm}$. This peak should be due to the aggregation. ${ }^{9}$ According to the exciton theory, this blue-shifted absorption is contributed by H-type aggregates, in which the dipoles are parallel. But we have known the structure of those dyes' LB films and that the area per chromophore is almost equal for $\mathrm{M}, \mathrm{B} 3$ and B5. Then why M has no absorption around $330 \mathrm{~nm}$ under the same film deposit condition? We assume that the 

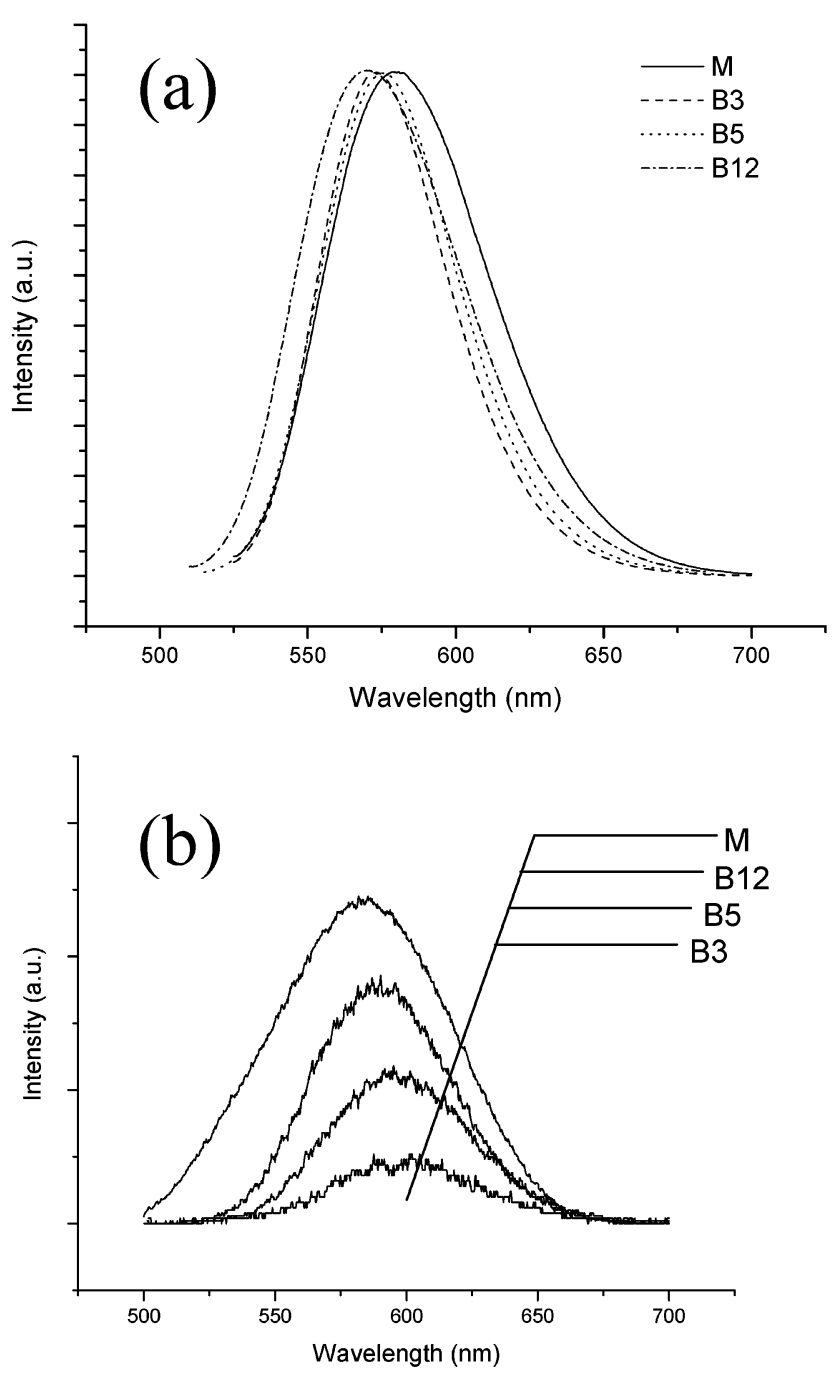

Figure 8. Fluorescence spectra of dyes. (a) chloroform solutions; (b) one-layer LB films on quartz substrates.

aggregate-like absorption peak is not the result of the real molecular aggregates but of the intramolecular interaction between the two chromophores in some dimers. There might be a very weak interaction between the two chromophores in one dimer molecule. This weak interaction might also be partially due to the exclusive volume effect induced by the linkage group in dimer species. However, the exclusive volume effect is not the most important factor because there are some reports ${ }^{21,22}$ on the intramolecular interaction of dimers whose linkage groups are similar to those of our system.

Another possibility for the difference between dimers and monomer is that the dimers might form excimers after excitation because their chromophores are close to each other. However, the results of fluorescence spectra, of both solution samples and LB films, show no evidence to support the occurrence of excimer fluorescence radiation in the longer-wave range (Figure 8). Usually, the excimer is formed in the dimers, which are linked by three-methylene groups,${ }^{29}$ the distance $(\sim 3 \AA)$ between those chromophores is very suitable for excimer formation. But as we know, B3 can hardly form a conformation in which there are parallel chromophores, so that the chromophores are not close enough to form a stable excimer; and the two chromophores in B5, although they are parallel, are also too far away ( $\sim 6 \AA$ ) from each other to form the excimer.

We have suggested that the slight absorption peak around $330 \mathrm{~nm}$ in dimers' LB films are due to the "intramolecular
TABLE 4: Results of RB3LYP/6-31G(d)//RHF/6-31G(d) Calculation of Dyes

\begin{tabular}{cccc}
\hline energy difference $(\mathrm{eV})$ & $\mathrm{B} 3$ & $\mathrm{~B} 5$ & $\mathrm{~B} 3 \mathrm{f}$ \\
\hline (LUMO+1)-(LUMO) & 0.093 & 0.032 & 0.394 \\
(HOMO)-(HOMO-1) & 0.039 & 0.014 & 0.160
\end{tabular}

TABLE 5: Photocurrent Generation of LB Films Modified ITO Electrodes

\begin{tabular}{lcccc}
\hline & M & B3 & B5 & B12 \\
\hline photocurrent $(\mu \mathrm{A})^{a}$ & 0.67 & 1.20 & 0.84 & 0.85 \\
quantum yield $(\%)$ & 0.31 & 0.63 & 0.47 & 0.31
\end{tabular}

${ }^{a}$ The photocurrent is measured under white light $\left(240 \mathrm{~mW} / \mathrm{cm}^{2}\right)$ irradiation, IR is filtered.

dipole-dipole exciton interaction", so we calculate (B3LYP/ 6-31G(d)//HF/6-31G(d)) the electronic structures of M, B3 and B5, using their molecular mechanical optimized conformations to find evidence for the dipole-dipole interaction. To investigate the electronic structure of the dimer when it is highly compressed in an LB film, we constructed a model by altering the dihedral angles of the methylene chain of B3 to a "U-shape" folded conformation, namely B3f (Figure 6(c)). Evidently, it is not stable in free conditions, but it may simulate the situation of some dimers in LB films, that is, two chromophores become much closer than those found in ordinary conditions.

It is found that the orbital numbers in $a$ dimers are twice that of a monomer, but the orbitals are not degenerate. Both B5 and B3 have two close HOMOs and two close LUMOs, i.e., there are energy difference between HOMO and HOMO-1 and between LUMO and LUMO+1 (Table 4). However, the HOMO and HOMO-1 have the same properties and they come from the same orbital of each chromophore in a dimer molecule. This is exactly caused by the dipole-dipole interaction, ${ }^{32,33,43,44}$ and it is an intramolecular interaction. But the energy gap between the splits in the B3 or B5 is so small that in chloroform we cannot find any evidence of the absorption shift in their spectra, indicating the weak coupling between the two chromophores in B3 or B5. As for the B3f conformation, it is a model that, to a certain degree, simulates the dimers in LB films; in that case, some molecules are compressed to have two extremely close chromophores. The calculation shows that both the energy gaps between HOMO and HOMO-1 and between LUMO and LUMO+1 are increased. Hence, a shifting absorption peak $(\sim 330 \mathrm{~nm})$ is detected in experiment. Also, the weak intensity of the absorption suggests that most of the molecules in LB films are not compressed as much as B3f, otherwise the absorption spectra of dimers would be extremely different from that of monomer and there would be some excimer-like fluorescence for the dimers' LB films. All of these electronic structure calculations indicate our previous assumptions of the LB films structure and of the absorption spectra are credible.

A typical three-electrode electrochemical cell is used to measure the photocurrent generated from the LB-monolayerfilm-modified ITO electrode. Because it is impossible to make any two LB film samples identical, at least eight individually prepared samples were employed in each experiment in order to get the mean value of the results. The mean photocurrent values are listed in Table 5. The external photoelectric conversion quantum yield (QY) is the criterion for the energy conversion potentiality, and it is calculated using eq 3

$$
Q Y=\frac{i_{\mathrm{ph}}}{e N_{\mathrm{p}}\left(1-A_{\lambda}\right)}
$$




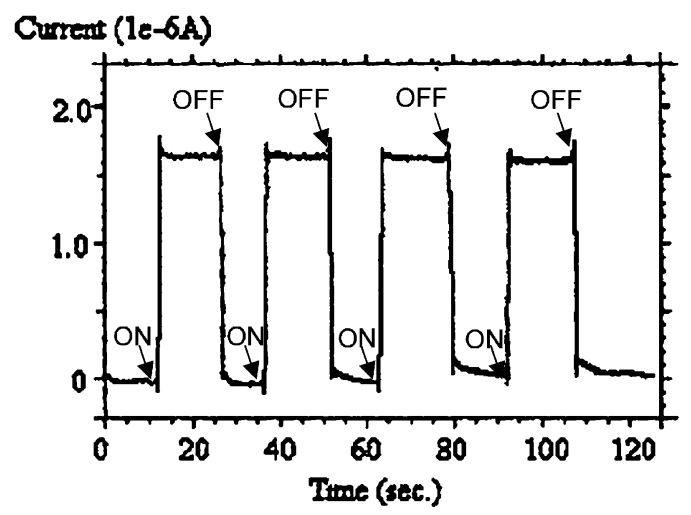

Figure 9. Typical photocurrent generated from B3 one-layer LB films modified ITO electrode. "ON" and "OFF" indicate the irradiation situations.

Here $i_{\mathrm{ph}}$ is the photocurrent generated from the working electrode when it is irradiated by the light with wavelength $\lambda$. $N_{\mathrm{p}}$ is the number of irradiating photons per second, and $\mathrm{A}_{\lambda}$ is the absorption percentage at wavelength $\lambda$.

From Table 5, we learn that the QY of B3 and B5 are larger than that of $\mathrm{M}$. Because the mean occupation area per chromophore of M, B3, and B5 are 40, 45, and $40 \mathrm{~nm}^{2}$, respectively, the dimerization will not influence the chromophore density of these LB films much. Therefore, the improvements of the quantum yields of B3 and B5 are not due to an increase in the number of the photoactive chromophores. If the photoelectric conversion efficiency of each chromophore is independent of the molecular structure or LB film structure, then the M, B3, and B5 LB films should have shown similar photocurrent generation and almost the same quantum yields. However, the remarkable difference between B3 (or B5) and M suggests that there must be some relationship between the photocurrent generation and the structure of the molecules. We sum up this phenomenon in a simple inequality " $1+1>2$ " because it seems that when a stilbazolium chromophore is linked with another one by suitable methylene groups, the phototcurrent generation is greater than twice that of the monomer.

At first, we changed some experimental conditions to find the mechanism of the current generation. Under normal conditions (the electrolyte solution is saturated with air and there is no additional electron donor or acceptor), the photocurrent is a cathodic one, indicating that the electron is ejected from the ITO electrode and flows through LB film and then transfers to the electrolyte. Figure 9 is a typical photocurrent response curve with the irradiation of white light from a xenon lamp; the current response is quite prompt.

The photocurrent action spectra of the M, B3, B5, and B12 are shown in Figure 10. Although the points, which are measured with a set of interference filters, are not continuous, it is evident that the shapes of these spectra coincide with corresponding LB films' absorption spectra (as Figure 7(b) shows, this absorption is not caused by aggregates but by individual molecules, see discussion before). This coincidence indicates that the photocurrent is generated from those molecules that are deposited on substrates, and it comes from the isolated molecules. If the intensity of irradiation light is increased, the photocurrent increases too.

We have published several papers on the PEC of similar derivatives before, and a key step in the mechanism of photocurrent generation is such that the chromophore is excited by light irradiation and produces an excited state with charge redistribution. It is well-known that chromophores like stilbazolium will form "charge-reversed" excited states. This charge

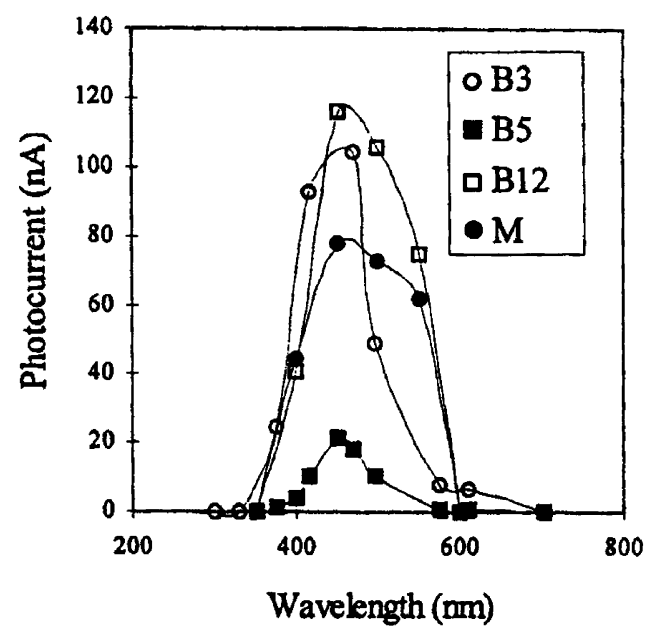

Figure 10. Action spectra of dyes. (solid square: M; open circle: B3; solid circle: B5; open square: B12)

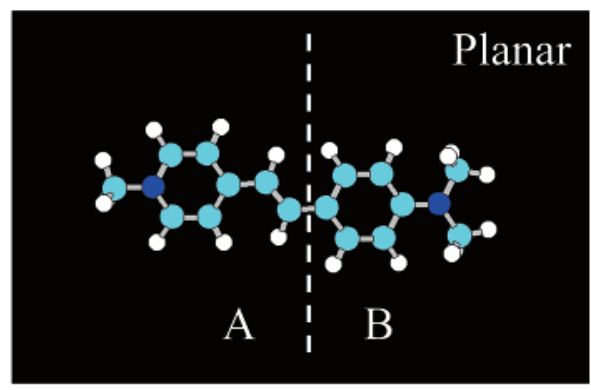

Charge Distribution

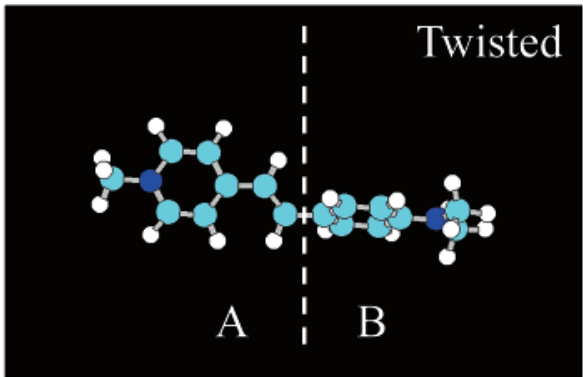

Excited State

A: 0.116

B: 0.884

(b)

Figure 11. Charge distribution calculation results of $M$. (a) planar conformation (optimized conformation for ground state); (b) twisted conformation (optimized conformation for excited state).

redistribution makes the excited state become an active site for electron exchange between the electrolyte solution and electrode. Here, we provide some concise results of our semiempirical calculations to accurately understand this view of point.

The charge distribution (MOPAC 6, PM3 Hamilton) was calculated with the result that the chromophore in dimers shows no difference from the monomer. Figure 11 shows the chromophore's charge distribution (PM3) and the effect of conformation change. Before excitation, the pyridinium moiety has the most positive charge (0.817) before excitation, and this causes strong static electric repulsion, which in turn separates the two chromophores in B3.

Calculations on the excited state of stilbazolium chromophore are also shown in Figure 11. The Franck-Condon (FC) state shows that the charge distribution is more uniform under light irradiation. The pyridinium moiety, the charge on which is more positive in the ground state, shares its positive charge with aminobenzene moiety. Even the polar direction is reversed: the aminobenzene moiety is more positively charged than the 


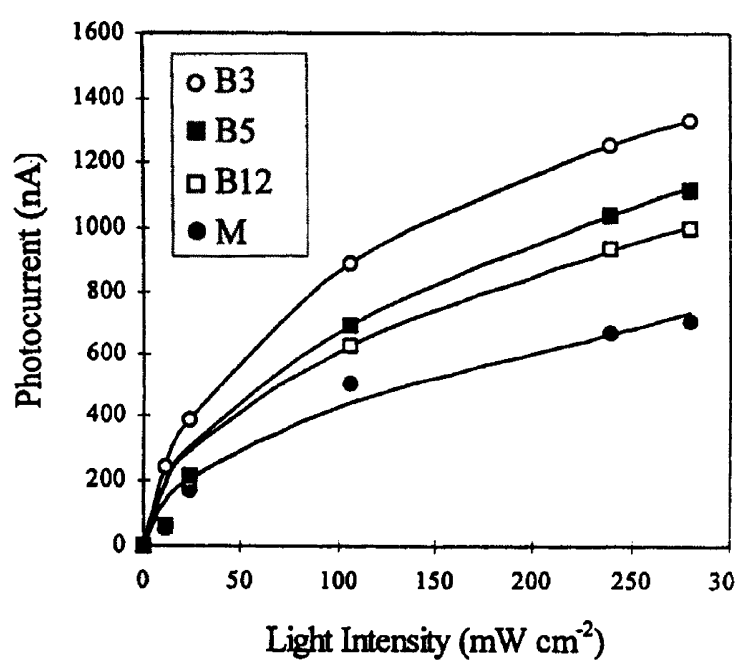

Figure 12. Effluence of light intensity to the photocurrent generation from the dye LB film modified ITO electrode. Zero bias, $0.5 \mathrm{~mol} \mathrm{dm}^{-3}$ $\mathrm{KCl}, 298 \mathrm{~K}$. (solid circle, M; open circle, B3; solid square, B5; open square, B12)

pyridinium does. The dipole moment of ground state is also greater than in the excited state, which is consistent with our spectroscopic results (the negative solvatochromic effect).

However, the FC state is not a stable conformation for the excited state and the semiempirical quantum mechanical optimization of stilbazolium's excited state gives a twisted state as shown in Figure 11(b). From the value of the charge distribution of this conformation, we can find that the twisted conformation not only stabilizes the excited state but also enhances the charge reversion of excited state, and of course, the twisted state has a greater dipole moment than the FC state for the enhanced charge separation.

Figure 12 shows the relationships between the photocurrent generation and irradiation light intensity of the dyes. Each curve is fitted well by a simple exponential eq 4 with suitable value of $k$-factor

$$
i=k I^{0.5}
$$

$I$ and $i$ represent, respectively, the intensity of irradiation light and the corresponding photocurrent. The relationship between the photocurrent generation and the irradiation light intensity suggests the fundamental character of the photoinduced electron transfer and recombination. ${ }^{36}$ The power exponent $(0.5)$ is the same for all the four dyes, indicating that the pathways of their photocurrent generation and recombination are similar, and that the photoactive processes of chromophores in dimers are quite similar to those in the monomer, that is to say, the dimerization makes no difference to the photocurrent generation mechanism. The same mechanism can be used in both monomer and dimer.

It is well-known that a bias voltage can change the photocurrent intensity in this photoelectric conversion system, and that a negative bias benefits the cathodic photocurrent. It is found that all the four dyes here follow this rule very well (Figure 13). This suggests once again that the electron is injected into the dyes from ITO electrode because the electric field caused by negative bias has the same polarity with the interfield at the ITO/LB surface. A negative bias accelerates the charge separation, assists the generation of the electron-hole pairs, reduces charge trapping during the transmission, and restrains the charge recombination. If the bias is positive, then the effects are reversed, and the photocurrent is decreased.

Usually, the photocurrent generation is strongly influenced by electron donors or acceptors in the electrolyte solution.

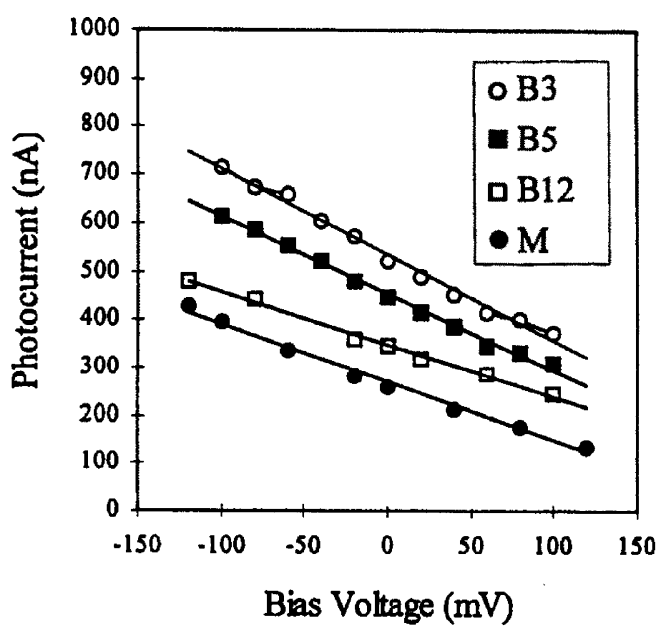

Figure 13. Effluence of bias on photocurrent generation from the dyeLB-film-modified ITO electrode. White light $\left(110 \mathrm{~mW} / \mathrm{cm}^{2}\right), 0.5 \mathrm{~mol}$ $\mathrm{dm}^{-3} \mathrm{KCl}, 298 \mathrm{~K}$. (solid circle: M; open circle: B3; solid square: B5; open square: B12)

Therefore, the investigation of such an effect helps us to better understand the photocurrent generation process. The donor is usually a compound with a low oxidation potential, so that it can easily lose an electron to the electrode; however, the compound with low reduction potential is a natural acceptor with strong electron-capturing ability.

Considering the solubility in electrolyte solution, we select hydroquinone $\left(\mathrm{H}_{2} \mathrm{Q}\right)$ and methyl viologen diiodide $\left(\mathrm{MVI}_{2}\right)$ as the electron donor and acceptor, respectively. ${ }^{24}$ The change in the photocurrent intensity caused by the electron donor/acceptor is significant. Because the four dyes show almost the same behaviors upon the introduction of donor/acceptor, only the details about B3 are listed here. The introduction of hydroquinone and methyl viologen diiodide leads to two opposite results. The irradiation light is white light $\left(240 \mathrm{~mW} / \mathrm{cm}^{2}\right)$ radiated from a Xenon lamp, the infrared light has been filtered out for protecting the electrode from heating and ruling out the thermoeffect. Before the addition of the donor/acceptor, the photocurrent is cathodic and stable, with a value of $1.25 \pm 0.05 \mu \mathrm{A}$.

Hydroquinone has an oxidation potential of $-4.61 \mathrm{eV}$ $(-0.13 \mathrm{~V}$ vs SCE) and, thus, easily gives away an electron to the electrode. It is found that the cathodic photocurrent is decreased when $\mathrm{H}_{2} \mathrm{Q}$ is gradually added into the electrolyte solution. And when the concentration of $\mathrm{H}_{2} \mathrm{Q}$ is greater than $1.25 \times 10^{-4} \mathrm{~mol} \mathrm{dm}^{-3}$, the photocurrent generated from electrode modified by B3 LB film is turned positive. Then the anodic photocurrent increases with the addition of $\mathrm{H}_{2} \mathrm{Q}$. When $\mathrm{H}_{2} \mathrm{Q}$ 's concentration achieves $2 \times 10^{-3} \mathrm{~mol} \mathrm{dm}^{-3}$, the positive photocurrent reaches its maximum $(\sim 1.0 \mu \mathrm{A})$.

Although methyl viologen cation $\left(\mathrm{MV}^{2+}\right)$ has its first reduction potential of $-4.51 \mathrm{eV}(-0.23 \mathrm{~V}$ vs SCE), it can capture the electron from the electrode effectively. There is no doubt that the existence of electron acceptor in the electrolyte solution accelerates the electron movement from electrode to electrolyte. Experimental results show an expected tendency of the photocurrent with the addition of $\mathrm{MV}^{2+}$ into electrolyte solution, and the cathodic photocurrent reaches its maximum (ca. $2.5 \mu \mathrm{A}$ ) when the concentration of $\mathrm{MV}^{2+}$ is greater than $1 \times 10^{-3} \mathrm{~mol}$ $\mathrm{dm}^{-3}$.

It is well-known that oxygen, which dissolved in electrolyte, is also a good electron acceptor in electrochemistry experiments, and that bubbling nitrogen gas into the electrolyte solution can drive oxygen out. Our experiments reveal that the oxygen also 


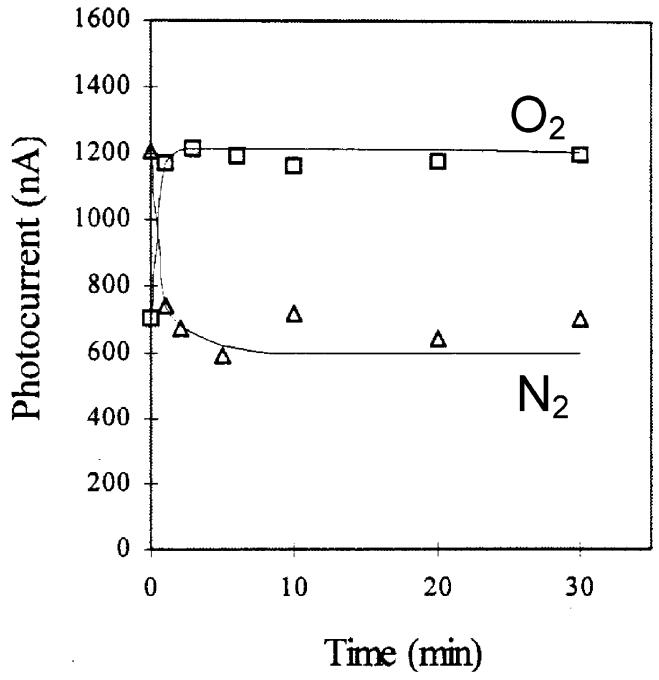

Figure 14. Effluence of the oxygen and nitrogen to the photocurrent generation from a B3-LB-film-modified ITO electrode. Zero bias, white light $\left(240 \mathrm{~mW} / \mathrm{cm}^{2}\right), 0.5 \mathrm{~mol} \mathrm{dm}{ }^{-3} \mathrm{KCl}, 298 \mathrm{~K}$.

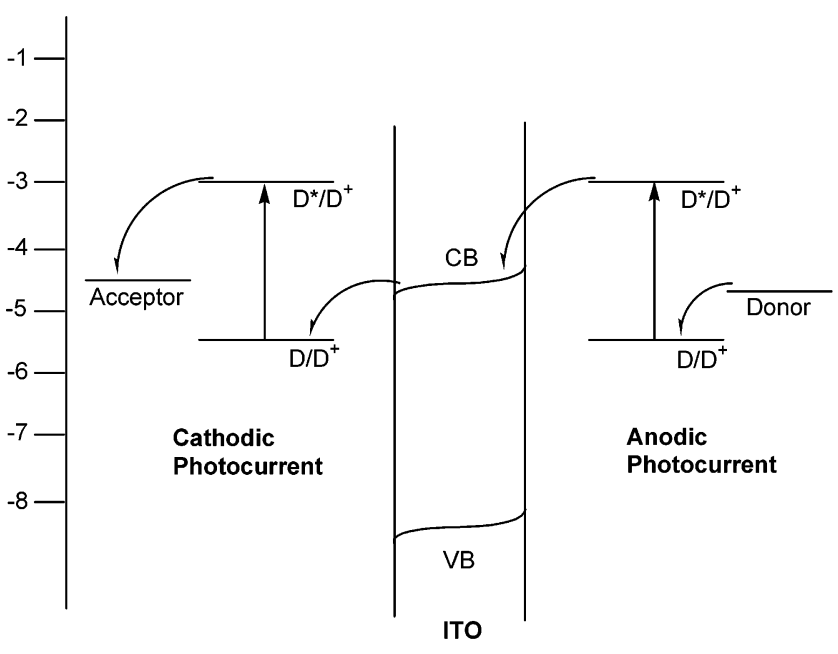

(a)

(b)

Figure 15. Schematic mechanism of photocurrent generation.

influence the photocurrent generation process. Figure 14 shows the relationship between the photocurrent and the bubbling time of the gas. Obviously, nitrogen restrains the cathodic photocurrent and reduces the value to around $600 \mathrm{nA}$. Then, if oxygen is bubbled into the nitrogen saturated electrolyte solution, the cathodic photocurrent is reconverted to $1200 \mathrm{nA}$.

In this way, we can propose the mechanism of the photocurrent generation as shown in Figure $15 . .^{24,44-46}$ In this figure, (a) represents the case of the cathodic photocurrent, whereas (b) represents the case of the anodic photocurrent. The energy level of the dye's ground state $(-5.42 \mathrm{eV})$ is calculated from M's first oxidation potential $(0.68 \mathrm{~V}$ vs SCE, and dimers show electrochemical inertia); the energy level of dye's excited state $(\sim-3 \mathrm{eV})$ is estimated by absorption spectrum. The energy levels of the ITO electrode's conduction and valence bands are around $-4.5 \mathrm{eV}$ and $-8.3 \mathrm{eV}$, respectively. Thus, a continuous cathodic photocurrent is generated. When there are electron acceptor molecules ( $\mathrm{MV}^{2+}$ or $\mathrm{O}_{2}$, etc.) in the electrolyte solution, they can obtain electrons from the dyes under irradiation. And the electrode donates relevant electrons from its conduction band to those dye molecules. When there are electron donor molecules $\left(\mathrm{H}_{2} \mathrm{Q}\right)$ in the electrolyte solution, they will replace the electrode's conduction band to donate electrons to dyes, and the electron finally arrives at the electrode's conduction band. This forms the anodic current.

From those detailed analyses of the photocurrent generation process and the spectroscopic properties, we can make a conclusion that the dimerization enhances the PEC efficiency when the linkage is suitable. However, the reason behind why the proper linkage benefits the PEC properties is still unclear. According to our assumption of the structure of LB films, the motility (vibration, twisting, etc.) of dimers will be much more difficult than that of the monomer. Although this difference has no effect on steady-state spectroscopic properties, it will probably influence the dynamical properties of molecules, both in ground state and in excited states. We do not have a definitive conclusion of the fundamental concepts behind the relationship between the dimers and the monomer, but there is a supposition in our minds that the difference is not the results of aggregation or excimer formation, and the energy transfer procedure might plays an important role here. Further photophysical experiments are now processing to explore the more convincing evidences to support our supposition.

\section{Conclusions}

(1) A series of stilbazolium dimers, in which the two chromophores are linked via variable methylene groups, has been designed and synthesized. Although the chemical structures of these dimers are center-symmetric (or quasi-center-symmetric), they can be folded in their LB films to form a noncentersymmetric orientation monolayer, which give the comparable SHG signals with the monomer. The surface pressure-area isotherms indicate the higher stability contributed from the dimerization. This is a new perspective that one may use external force to make a molecule to change its conformation to fit one's needs.

(2) B3 and B5 have higher photoelectric conversion quantum yields than M. Both steady-state absorption and fluorescence experiments indicate that no strong intramolecular interaction exists in this system, and some computational results also imply that the common intramolecular dipole-dipole interaction between the two chromophores in dimer molecules seldom occur in these stilbazolium dimers. This is due to the static repulsion between the two closer positive charged sites of the molecule. Thus, we suppose that the enhanced photocurrent generation of dimerization probably comes from the structural effect caused by dimerization, which might influence the energy transfer procedure.

(3) According to absorption spectra, SHG signals and computational results, the three-methylene linkage is not long enough for two stilbazolium chromophores to take parallel orientation. And in the LB films, B5 has a U-shape, whereas $\mathrm{B} 3$ has a V-shape conformation. The crystal diffraction result of B5 also partially supports this conclusion.

\section{Experimental Section}

1. Synthesis. $N$-methyl, $N$-octadecylaniline. $9.88 \mathrm{~g}(0.092 \mathrm{~mol}$, $10 \mathrm{~mL}) \mathrm{N}$-methylaniline reacted with $35.06 \mathrm{~g}(0.092 \mathrm{~mol})$ octadecyl iodide in a mixture of $30 \mathrm{~mL}$ toluene and $50 \mathrm{~mL} 30 \%$ sodium hydroxide aqueous solution containing $0.19 \mathrm{~g} \mathrm{~N}$ hexadecyl, $N, N, N$-trimethylammonium bromide (phase transfer catalyst) at $80{ }^{\circ} \mathrm{C}$ under stirring for at least 7 days. The toluene phase was washed with sodium chloride solution and then evaporated. The residue was purified on a silica gel column with pure petroleum ether and then with petroleum ether-ethyl acetate mixture as an eluent. Yield: $90 \%$. 
$N$-methyl,N-octadecylaminobenzaldehyde. $14.36 \mathrm{~g}$ (0.04 mol) $\mathrm{N}$-methyl,N-octadecylaniline was added to $70 \mathrm{~mL} \mathrm{DMF}$ (freshly distilled). After the mixture was cooled to $4{ }^{\circ} \mathrm{C}, 5 \mathrm{~mL} \mathrm{POCl}_{3}$ was added dropwise to the mixture. This resulting mixture was kept stirred at room temperature for $1 \mathrm{~h}$ and then at $100{ }^{\circ} \mathrm{C}$ for $4 \mathrm{~h}$. The reactant was purified on silica gel column after being neutralized to $\mathrm{pH} 7$ with sodium hydroxide solution. $12 \mathrm{~g}$ target product was obtained. Yield: $77.5 \%, \mathrm{Mp}: 51{ }^{\circ} \mathrm{C}$.

1,n-Bis [4-methylpyridinyl]alkane(propane, pentane, dodecane) dibromide. $0.12 \mathrm{~mol}$ of 4-methylpyridine and $0.04 \mathrm{~mol}$ of 1, $n$-dibromoalkane $(n=3$, propane; $n=5$, pentane; $n=12$, dodecane) were added to $70 \mathrm{~mL}$ anhydrous ethanol. The mixture was refluxed for $7 \mathrm{~d}$. The product was obtained after evaporation of solvent and then recrystallized in a mixture solvent of ethanol and ether. Yield: sustituted propane, $82 \%$; substituted pentane, 85\%; substituted dodecane, $87 \%$. Mp: substituted propane, dec $\sim 215^{\circ} \mathrm{C}$; substituted pentane, $218 \sim 220{ }^{\circ} \mathrm{C}$ (dec partly at $\sim 210$ $\left.{ }^{\circ} \mathrm{C}\right)$; substituted dodecane, $117^{\circ} \mathrm{C}$.

1,n-Bis [(E)- 4-(2-(4-(N-methyl, N-octadecylamino) phenyl) ethenyl) pyridinyl]-alkane dibromide $(n=3$, alkane $=$ propane, B3; $n=5$, alkane = pentane, B5; $n=12$, alkane = dodecane, B12). The mixture of 1,n-Bis [4-methylpyridinyl]alkane dibromide and $N$-methyl, $N$-octadecylbenzaldehyde (molar ratio 1:3) was dissolved in anhydrous ethanol containing piperidine as catalyst and was kept refluxing for $4 \mathrm{~h}$. After the evaporation of the solvent, the residue was purified on a silica gel column.

[(E)-N-methyl-4-(2-(4-(N-methyl, N-octadecylamino) phenyl) ethenyl) pyridinium] iodide $(M)$. The mixture of $193.5 \mathrm{mg}(0.5$ mmol) N-octadecyl, $N$-methylamino- benzaldehyde, $117.5 \mathrm{mg}$ (0.5 mmol) 1,4 dimethylpyridinium Iodide, $0.2 \mathrm{~mL}$ piperidine and $20 \mathrm{~mL}$ ethanol was refluxed for $6 \mathrm{~h}$. The resulting system was cooled, and the precipitate was collected. Recrystallization was done twice with ethanol.

1, n-Bis [(E)-4-(2-(4-(N,N-dimethyl)phenyl)ethenyl)pyridinyl]alkane dibromide $\left(n=3\right.$, alkane = propane, $B 3^{\prime} ; n=5$, alkane $=$ pentane, $B 5^{\prime} ; n=12$, alkane = dodecane, B12'). The synthetic procedures are similar to the $\mathrm{BC} 3, \mathrm{BC} 5$, and $\mathrm{BC} 12$. $\mathrm{B} 3^{\prime}, \mathrm{B}^{\prime}, \mathrm{B} 12^{\prime}$, and $\mathrm{M}^{\prime}$ were synthesized for improving the solubility in some high polar slovents.

2. Spectroscopic Measurement. The UV-vis absorption spectra of B3, B5, B12, and M in solutions and LB films on quartz slides were recorded by a Shimadzu UV-3100 Spectrophotometer, with blank chloroform (A.R grade) or a quartz slide as a reference, respectively. Static-state fluorescence excitation and emission spectra were measured by a Hitachi F4500 fluorescence spectrophotometer, using proper excitation cutoff filters. Excitation and emission bandwidths of $5 \mathrm{~nm}$ were used, and the fluorescence spectra were corrected for nonlinear instrumental response and blank solvents.

3. Surface Pressure-Area Isotherm and Film Deposition. A computer-controlled 622 Nima Langmuir trough (Nima Co., England) was used for $\pi-A$ isotherm measurements and monolayer deposition. The surface pressure-area per molecule $(\pi-A)$ isotherm was obtained by compressing the spreading sample, which was added dropwise from a micro-injector onto deionized water (18.0 M $\Omega \mathrm{cm}, \mathrm{pH}$ 5.6) surface, at a barrier speed of $50 \mathrm{~cm}^{2} / \mathrm{min}$. The LB trough was controlled at $20 \pm$ $0.5^{\circ} \mathrm{C}$. Monolayers of B3, B5, B12, or M were obtained by spreading the four corresponding chloroform solutions $(0.44 \mathrm{mg}$ $\mathrm{ml}^{-1}$ for B3, $0.54 \mathrm{mg} \mathrm{ml}^{-1}$ for B5, $0.68 \mathrm{mg} \mathrm{ml}^{-1}$ for B12, 0.54 $\mathrm{mg} \mathrm{ml}^{-1}$ for $\mathrm{M}$ ) onto the water surface. The floating layer was compressed to a surface pressure of $30 \mathrm{mN} / \mathrm{m}$ for each of B3, B5, B12 or M. The compressed floating layer was kept at this pressure during the deposition procedure, the monolayer was transferred at a speed of $5 \mathrm{~mm} / \mathrm{min}$ onto the hydrophilically pretreated quartz or transparent ITO glass $(\sim 50 \Omega / \square)$ substrates in upstroke. The transfer ratio were $1.0 \pm 0.1$.

4. Photoelectrochemical Measurements. The conventional three-electrode setup with a $0.8 \mathrm{~cm}^{2}$ effective contact area was used in all irradiation experiments. This experiment used ITO glass slides modified by the monolayer LB films as working electrode (WE), a platinum electrode as the counter-electrode (CE), a SCE as the reference electrode (RE), and a $0.5 \mathrm{~mol}$ $\mathrm{dm}^{-3} \mathrm{KCl}$ aqueous solution as supporting electrolyte solution in photocurrent generation measurements. All measurements were carried out at $25 \pm 1{ }^{\circ} \mathrm{C}$ on a Model 600 Voltametric Analyzer (CH Instruments, USA). The excitation source for the photochemical experiments was a $500 \mathrm{~W}$ Xenon Lamp (Ushio Electric, Japan). The incident white light was perpendicular to the contact area of the working electrode. The intensity of the incident beam was checked by a model LM-91 Photopower meter (National Institute of Metrology, Beijing, China). Different wavelengths were obtained by using a series of interference filters (Toshiba, Japan) with certain band-passes. IR light was filtered out by a Toshiba IRA-25S filter (Toshiba Co., Japan) in all experiments to avoid thermal effects. Photocurrents were the average values of at least six independent measurements.

5. SHG Measurement. The SHG signals of the LB films of $\mathrm{B} 3, \mathrm{~B} 5, \mathrm{~B} 12$, and $\mathrm{M}$ were measured in transmission with a Y-cut quartz plate as a reference $\left(d_{11}=1.2 \times 10^{-9} \mathrm{esu}\right)$. The intensity of the incident Q-switch Nd:YAG laser (GCR-4, $1064 \mathrm{~nm}$, Spectra-Physics) was $140 \mathrm{~mW} \mathrm{~cm}^{-2}$, pulse duration was $10 \mathrm{~ns}$ and repetition rate was $10 \mathrm{~Hz}$. A $1 / 2 \lambda$ plate and a Glan-Taylor polarizer were used to vary the polarization direction of the laser beam. The linearly polarized laser beam was directed at an incident angle of $45^{\circ}$ onto the vertically mounted sample. The output beam was filtered and monochromated, and then the signal was recorded by a digital oscilloscope (HP 54510A). The second harmonic intensities $\left(I_{2 \omega}\right)$ obtained from the monolayer LB film were analyzed by the general procedure described by Ashwell. ${ }^{35}$

6. X-ray Crystal Structure Determination. A dark red color crystal of B5' with dimensions $0.60 \times 0.40 \times 0.30 \mathrm{~mm}^{3}$ was selected for structure determination. It was mounted on a Rigaku AFC65 diffractometer with highly oriented graphite crystal monochromated $\mathrm{MoK} \alpha$ radiation. The reflection data were collected in the range $2 \theta 4.0 \sim 40.0^{\circ}$. The cell parameters were calculated by the least-squares method; 2406 independent reflections were collected, from which 862 reflections with $F$ $>4.0 \sigma(\mathrm{F})$ were used in the refinement. Intensities were corrected. The final $R$ and $R_{\mathrm{w}}$ were 0.095 and 0.076 , respectively.

7. Computational Chemistry. A conformation search was carried out by a SGI $\mathrm{O}_{2}$ workstation using a Cerious ${ }^{2}$ software package and cvff95 force field. Semiempirical quantum chemical calculations were carried out by MOPAC 6.0 or HyperChem5.1 by means of an Intel Pentium workstation. The PRECISE option was used as a convergence criterion for calculation of geometry for all MOPAC calculations. DFT calculations were operated by Gaussian 98 on a Linux based workstation. PM3 hamilton was used for semiempirical calculations, and for more precise calculations, such as molecular energy levels, B3LYP/6-31G(d)//RHF/6-31G(d) was performed. In semiempirical calculations, a configuration interaction $(\mathrm{CI})$ related to 10 energy levels was taken into considered.

Acknowledgment. The authors thank the State Key Project of Fundamental Research (Grant Nos. G1998061308 and 
2001CCD04300), National Natural Science Foundation of China (20023005) and Doctoral Program Foundation of Higher Education (9900132) for financial support.

Supporting Information Available: The detailed data of synthesis and crystal structure determination. This material is available free of charge via the Internet at http://pubs.acs.org.

\section{References and Notes}

(1) Ephardt, H.; Fromherz, P. J. Phys. Chem. 1989, 93, 7717.

(2) Rocker, C.; Heilemann, A.; Fromherz, P. J. Phys. Chem. 1996, 100, 12172 .

(3) Song, Q.; Bohn, P. W.; Blanchard, G. J. J. Phys. Chem. 1997, 101, 8865.

(4) Kim, O. K.; Choi, L. S.; Zhang, H. Y.; He, X. H.; Shih, Y. H. J. Am. Chem. Soc. 1996, 118, 12220.

(5) Evans, C. E.; Bohn, P. W. J. Am. Chem. Soc. 1993, 115, 3306. 93,73

6) Song, Q.; Xu, Z.; Lu, W.; Bohn, P. W. Colloid and Surface 1994

(7) Narang, U.; Zhao, C. F.; Bhawalkar, J. D.; Bright, F. V.; Prasad,

P. N. J. Phys. Chem. 1996, 100, 4521.

(8) Zhao, C. F.; Gvishi, R.; Narang, U.; Ruland, G.; Prasad, P. N. J. Phys. Chem. 1996, 100, 4526.

(9) Evans, C. E.; Song, Q.; Bohn, P. W. J. Phys. Chem. 1993, 97, 12302.

(10) He, G. S.; Kim, K. S.; Yuan, L.; Cheng, N.; Prasad, P. N. Appl. Phys. Lett. 1997, 71, 1619.

(11) Cao, X.; Tolbert, R. W.; McHale, J. L.; Edwards, W. D. J. Phys. Chem. A 1998, 102, 2739.

(12) Kim, J.; Lee, M. J. Phys. Chem. A 1999, 103, 3378.

(13) Lusk, A. L.; Bohn, P. W. J. Phys. Chem. B 2001, 105, 462.

(14) Wandelt, B.; Turkewitsch, P.; Stranix, B. R.; Darling, G. D J. Chem. Soc., Faraday Trans. 1995, 91, 4199.

(15) Ashwell, G. J.; Walker, T. W.; Leeson, P.; Grummt, U. W.; Lehmann, F. Langmuir 1998, 14, 1525.

(16) Lang, A. D.; Zhai, J.; Huang, C. H.; Gan, L. B.; Zhao, Y. L.; Zhou, D. J.; Chen, Z. D. J. Phys. Chem. B 1998, 102, 1424.

(17) Jones, M. A.; Bohn, P. W. Anal. Chem. 2000, 72, 3776.

(18) Song, X.; Perlstein, J.; Whitten, D. G. J. Am. Chem. Soc. 1995 , $117,7816$.

(19) Furman, I.; Geiger, H. C.; Whitten, D. G. Langmuir 1994, 10, 837

(20) Chambers, R. W.; Kajiwara, T.; Kearns, D. R. J. Phys. Chem. 1974, 78,380 .

(21) Lu, L.; Lachicotte, R. J.; Penner, T. L.; Perlstein, J.; Whitten, D. G. J. Am. Chem. Soc. 1999, 121, 8146.
(22) Zeena, S.; Thomas, K. G. J. Am. Chem. Soc. 2001, 123, 7859.

(23) Bartholomew, G. P.; Bazan, G. C. Acc. Chem. Res. 2001, 34, 30

(24) Wu, D. G.; Huang C. H.; Gan, L. B.; Zhang, W.; Zheng J.; Luo,

H. X.; Li, N. Q. J. Phys. Chem. B 1999, 103, 4377.

(25) Wu, D. G.; Huang, C. H.; Huang, Y.; Gan, L. B.; Yu, A. C.; Ying,

L. M.; Zhao, X. S. J. Phys. Chem. B 1999, 103, 7130.

(26) Li, F. Y.; Zheng, J.; Huang, C. H.; Jin L.; Haung Y.; Gao, J.; Liu, T.; Zhao, X. Appl. Surf. Sci. 2000, 161, 178. and references therein.

(27) Li, F.; Huang, Y.; Huang, C.-H.; Zheng, J.; Guo, J. J. Mater. Chem.

2001, 11, 1783 .

(28) Wang, Z. S.; Li, F. Y.; Huang, C. H.; Wang, L.; Wei, M.; Jin, L.

P.; Li, N. Q. J. Phys. Chem. B 2000, 104, 9676.

(29) Hirayama, F. J. Chem. Phys. 1965, 42, 3163.

(30) Ashwell, G. J.; Jackson, P. D.; Crossland, W. A. Nature 1994, 368 438 .

(31) Ashwell, G. J.; Jetteries, G.; Hamilton, D. G.; Lynch, D. E.; Roberts,

M. P. S.; Bahra, S.; Brown, C. R. Nature 1995, 375, 385.

(32) Corder, C. N.; Way, J. L. J. Med. Chem. 1966, 9, 638.

(33) Mishra, J. K.; Behera, P. K.; Parida, S. K.; Mishra, B. K. Indian J. Chem. 1992, 31B, 118 .

(34) Heinz, T. F.; Chen, C. K.; Richard, D.; Shen, Y. R. Phys. Rev Lett. 1982, 48, 478.

(35) Heinz, T. F.; Tom, H. W. K.; Shen, Y. R. Phys. Rev. A 1983, 28, 1883.

(36) Bubeck, C.; Laschewsky, A.; Lupo, D.; Neher, D.; Ottenbreit, P.;

Paulus, W.; Prass, W.; Ringsdorf, H.; Wegner, G. Adv. Mater. 1991, 3, 54

(37) Oudar, J. L. J. Chem. Phys. 1977, 67, 446.

(38) Oudar, J. L.; Chemla, D. S. J. Chem. Phys. 1977, 66, 2664.

(39) Evans, C. E.; Bohn, P. W. J. Am. Chem. Soc. 1993, 115, 3306.

(40) Carpenter, M. A.; Willand, C. S.; Penner, T. L.; William, D. J.; Mukanmel, S. J. Phys. Chem. 1992, 96, 2801.

(41) Girling, I. R.; Cade, N. A.; Kolinsky, P. V.; Jones, R. J.; Peterson, I. R.; Ahmed, M. M.; Neal, D. B.; Petty, M. C. Roberts, G. G.; Feast, W. J. J. Opt. Soc. Am. B 1987, 4, 950.

(42) Schildkraut, J. S.; Penner, T. L.; Willand, C. S.; Ulman, A. Opt. Lett. 1998, 13, 134 .

(43) Cornil, J.; Beljonne, D.; Calbert, J.-P.; Bredas, J.-L. Adv. Mater 2001, 13, 1053

(44) Xia, W. S.; Huang, C. H.; Ye, X. Z.; Luo, C. P.; Gan, L. B.; Liu, Z. F. J. Phys. Chem. 1996, 100, 2244

(45) Leblanc, R. M.; Blanchet, P.-F.; Cote, D.; Gugeshashvili, M. I.; Munger, G.; Volkov, A. G. "Light energy conversion with pheophytim a chlorophyll a monolayers at the optical transparent electrode", Photochemistry and Photoelectrochemistry of Organic and Inorganic Molecular Thin Films, Lawrence, M. F.; Frank, A. J.; Ramasesha, S.; Wamser, C. C. Eds. Proc. SPIE 1991, 1436, 92.

(46) Rettig, W. Top. Curr. Chem. 1994, 169, 253. 